\title{
Reduced and irreducible simple algebraic extensions of commutative rings
}

\author{
S.V. Mihovski
}

\begin{abstract}
Let $A$ be a commutative ring with identity and $\alpha$ be an algebraic element over $A$. We give necessary and sufficient conditions under which the simple algebraic extension $A[\alpha]$ is without nilpotent or without idempotent elements.
\end{abstract}

\section{INTRODUCTION}

Let $A$ be a commutative ring with identity element 1 . We shall say that $K$ is a commutative ring extension of $A$, or $A$ is a subring of $K$, if $A$ and $K$ are commutative rings with common identity element and $A \subseteq K$.

Suppose that $K$ is a commutative ring extension of $A$ and let $\alpha \in K$ be an algebraic element over $A$. If $\alpha$ is a root of a nonzero polynomial $f(x) \in A[x]$ of a minimal degree $n$, then we shall say that $f(x)$ is a minimal polynomial of $\alpha$ over the ring $A$. The intersection of all subrings of $K$, containing $A$ and $\alpha$, we shall denoted by $A[\alpha]$. The ring $A[\alpha]$ is called a simple algebraic extension of $A$, which is obtained by adjoining $\alpha$ to $A$.

Let $f(x)$ be any nonzero polynomial over the ring $A$. If the leading coefficient of $f(x)$ is $a_{0}=1$, then $f(x)$ is said to be a monic polynomial over $A$. And what is more, if the leading coefficient $a_{0}$ of $f(x)$ is a regular element in $A$, i.e. $a_{0}$ is not zero divisor in $A$, then we shall say that $f(x)$ is a regular polynomial over the ring $A$.

Recall that the ring $A$ is called a reduced ring if $A$ has no nonzero nilpotent elements. The $\operatorname{ring} A$ is said to be irreducible if $A$ has no nontrivial idempotents.

A main result in [11] asserts that if $A$ is a reduced commutative ring, $f(x)$ is a monic minimal polynomial of $\alpha$ over $A$ and the discriminant $\Delta(f)$ is a regular element in $A$, then the simple algebraic extension $A[\alpha]$ is a reduced ring. Also in [11] is proved that if $A$ is an irreducible ring and the minimal polynomial $f(x)$ of $\alpha$ is monic and irreducible over $A$, then the simple algebraic extension $A[\alpha]$ again is irreducible. So here arises the

2010 Mathematics Subject Classification. 13B25, 13B02.

Key words and phrases. Commutative rings, Polynomials, Discriminants, Resultants, Simple algebraic extensions, Reduced rings, Irreducible rings. 
problem to find necessary and sufficient conditions under which the ring $A[\alpha]$ is reduced or irreducible. In this paper we solve these two problems in the parts 3 and 4 , respectively.

\section{Preliminary lemmas and definitions}

It is well known that every polynomial $f(x)$ of degree $n$ with coefficients from a field $F$ has at most $n$ roots in every field extension of $F$. Moreover, there exists a field extension $\bar{F} \supseteq F$ such that $\bar{F}$ contains exactly $n$ roots of $f(x)$. But this fact does not hold for the ring extensions of $F$. For example, let $G$ be a direct product of $m \geq 2$ cyclic groups of order $n$ and let $K=F G$ be the group ring of the group $G$ over the field $F$. Then $K$ is a ring extension of $F$ and every element of $G$ is a root of the polynomial $f(x)=x^{n}-1 \in F[x]$. Thus $f(x)$ has at least $n^{m}$ roots in $K$.

Let

$$
f(x)=a_{0} x^{n}+a_{1} x^{n-1}+\cdots+a_{n-1} x+a_{n} \quad(n \geq 1)
$$

be a polynomial over $A$ of degree $n$. We shall say that the elements $\alpha_{1}, \alpha_{2}$, $\ldots, \alpha_{n}$ form a canonical system roots of the polynomial $f(x) \in A[x]$ if there exists a commutative ring extension $K \supseteq A$ such that $\alpha_{1}, \alpha_{2}, \ldots, \alpha_{n} \in K$ and

$$
f(x)=a_{0}\left(x-\alpha_{1}\right)\left(x-\alpha_{2}\right) \cdots\left(x-\alpha_{n}\right) .
$$

Every regular polynomial over $A$ has at last one canonical system roots [9]. But example shows that over some rings $A$ there exist polynomials that have not roots. Moreover, there exist polynomials which have roots, but they have not canonical systems of roots. For more details see [9].

Later on we shall use the following two definitions. A discriminant of the polynomial (1) we shall call the following determinant of order $2 n-1$

$$
\Delta(f)=\varepsilon\left|\begin{array}{cccccccc}
1 & a_{1} & \ldots & a_{n} & 0 & 0 & \ldots & 0 \\
0 & a_{0} & a_{1} & \ldots & a_{n} & 0 & \ldots & 0 \\
\dddot{0} & \dddot{0} & \ldots & \ldots & \ldots & \ldots & \ldots & \ldots \\
n & (n-1) a_{1} & (n-2) a_{2} & \ldots & a_{n-1} & 0 & \ldots & 0 \\
0 & n a_{0} & (n-1) a_{1} & \ldots & 2 a_{n-2} & a_{n-1} & \ldots & 0 \\
\ldots & \ldots & \ldots & \ldots & \ldots & \ldots & \ldots & \ldots \\
0 & 0 & \ldots & 0 & n a_{0} & (n-1) a_{1} & \ldots & a_{n-1}
\end{array}\right|,
$$

where $\varepsilon=(-1)^{\frac{n(n-1)}{2}}$. So, for $n=1$ and $n=2$ we have $\Delta(f)=1$ and $\Delta(f)=a_{1}^{2}-4 a_{0} a_{2}$, respectively. Let

$$
g(x)=b_{0} x^{m}+b_{1} x^{m-1}+\cdots+b_{m-1} x+b_{m} \quad(m \geq 1)
$$

be another polynomial over $A$. Then the determinant of order $n+m$ 


$$
R(f, g)=\left|\begin{array}{cccccccc}
a_{0} & a_{1} & \ldots & a_{n} & 0 & \ldots & 0 & 0 \\
0 & a_{0} & a_{1} & \ldots & a_{n} & 0 & \ldots & 0 \\
\ldots & \ldots & \ldots & \ldots & \ldots & \ldots & \ldots & \ldots \\
0 & 0 & \ldots & a_{0} & a_{1} & \ldots & a_{n-1} & a_{n} \\
b_{0} & b_{1} & \ldots & b_{m} & 0 & \ldots & 0 & 0 \\
0 & b_{0} & b_{1} & \ldots & b_{m} & 0 & \ldots & 0 \\
\ldots & \ldots & \ldots & \ldots & \ldots & \ldots & \ldots & \ldots \\
0 & 0 & \ldots & b_{0} & b_{1} & \ldots & b_{m-1} & b_{m}
\end{array}\right|
$$

is said to be a resultant of the polynomials $f(x)$ and $g(x)$ (see $[3,8,13])$.

If $e_{1}, e_{2}, \ldots, e_{k}$ is a full orthogonal system idempotents of the ring $A$, that is the $\operatorname{ring} A$ is a direct sum of the ideals $e_{i} A \quad(i=1, \ldots, k)$, then for the polynomials $f(x), g(x) \in A[x]$ we put $f_{i}(x)=e_{i} f(x)$ and $g_{i}(x)=e_{i} g(x)$. So from the definition of $R(f, g)$ we conclude that

Likewise,

$$
R(f, g)=R\left(f_{1}, g_{1}\right)+R\left(f_{2}, g_{2}\right)+\cdots+R\left(f_{k}, g_{k}\right)
$$

$$
\Delta(f)=\Delta\left(f_{1}\right)+\Delta\left(f_{2}\right)+\cdots+\Delta\left(f_{k}\right) .
$$

Later on we shall use these facts without special stipulations.

Let $\alpha_{1}, \alpha_{2}, \ldots, \alpha_{n}$ be a canonical system roots of the polynomial (1). In [9] it is proved that

$$
R(f, g)=a_{0}^{m} g\left(\alpha_{1}\right) g\left(\alpha_{2}\right) \cdots g\left(\alpha_{n}\right)
$$

for every polynomial $g(x) \in A[x]$, even when $g(x)$ does not have roots. Moreover, if $n \geq 2$ and $f^{\prime}(x)$ is the prime derivative of $f(x)$, then [10]

$$
\Delta(f)=(-1)^{\frac{n(n-1)}{2}} \cdot a_{0}^{n-2} \cdot f^{\prime}\left(\alpha_{1}\right) \cdot f^{\prime}\left(\alpha_{2}\right) \cdots f^{\prime}\left(\alpha_{n}\right) .
$$

When $A$ is a field, then (4) and (5) show the well known facts that $R(f, g)=0$ if and only if $f(x)$ and $g(x)$ have common roots and $f(x)$ has multiple roots if and only if $\Delta(f)=0$. For arbitrary polynomials $f(x), g(x) \in A[x]$ we have the following.

Lemma 1. (see [9], also [8] p.159 and [13] p. 130) Let $A$ be any commutative ring with identity. If (1) and (3) are polynomials over $A$, then

(i) There exist polynomials $\varphi(x), \psi(x) \in A[x]$ such that $\operatorname{deg} \varphi(x) \leq m-$ $1, \operatorname{deg} \psi(x) \leq n-1$ and

$$
R(f, g)=\varphi(x) f(x)+\psi(x) g(x) .
$$

(ii) If $n \geq 2$, then there exist polynomials $u(x), v(x) \in A[x]$ such that $\operatorname{deg} u(x) \leq n-2, \operatorname{deg} v(x) \leq n-1$ and

$$
\Delta(f)=u(x) f(x)+v(x) f^{\prime}(x) .
$$

So we obtain

Corollary 1. [9] Let $f(x)$ and $g(x)$ be polynomials over the ring $A$.

(i) If $f(x)$ and $g(x)$ have common roots, then $R(f, g)=0$.

(ii) If $f(x)$ has multiple roots, then $\Delta(f)=0$. 
Examples show that the converse statements of the preceding corollary in the general case are erroneous [9].

Let $S$ be a multiplicatively closed set of regular elements in $A$, that is $S$ contains the product of every his two elements and every element of $S$ is not zero divisor in $A$. Then there exists a ring of quotients $S^{-1} A$ with respect to $S$ ([12], p. 146). Every element of $S^{-1} A$ is of the form $s^{-1} a$, where $s \in S$, $a \in A$. Thus all elements of $S$ are invertible in $S^{-1} A$. If $S$ is the set of all regular elements in $A$, then $S^{-1} A$ is said to be the classical ring of quotients, that we shall denote by $Q(A)$.

Lemma 2. Let $A$ be a ring with identity and $f(x)$ be a regular polynomial over $A$ of degree $n \geq 1$. Then there exists a ring extension $K \supseteq A$ such that $f(x)$ is a minimal polynomial over $A$ of some element $\alpha \in K$.

Proof. Let (1) be a regular polynomial over $A$. First, suppose that $a_{0}=1$. If $\operatorname{deg} f(x)=n=1$, then we put $\alpha=-a_{1}$ and the statements are trivial. If $n \geq 2$, then let $K=A[y] / I$ be the quotient ring of the polynomial ring $A[y]$ modulo the principal ideal $I$, generated by the polynomial $f(y)$. Thus $\bar{A}=\{a+I \mid a \in A\}$ is a subring of $K$ and, because $a_{0}=1$, it is clear that $A$ and $\bar{A}$ are isomorphic rings. So $A$ can be viewed as a subring of $K$. Obviously, $f(x)$ is a minimal polynomial of the element $\alpha=y+I \in K$.

If $a_{0} \neq 1$, then let $P=Q(A)$ be the classical ring of quotients of $A$. Now $A \subseteq P, f(x) \in P[x]$ and $a_{0}$ is an invertible element of $P$. Therefore $g(x)=a_{0}^{-1} f(x)$ is a monic polynomial over $P$ and the statement for $g(x)$ holds. Thus we conclude that there exists a commutative ring extension $K \supseteq P$ such that $g(x)$ is a minimal polynomial over $P$ of some element $\alpha \in K$. Then it is clear that $f(x)=a_{0} g(x)$ is a minimal polynomial of $\alpha$ over $A$, as was to be shoved.

When $\alpha \in K$ and $P=Q(A) \subseteq K$, then by definition there exists the simple ring extensions $P[\alpha]$ with $A[\alpha] \subseteq P[\alpha]$. But, if $P \not \subset K$, then $P[\alpha]$ is not defined in the general case. Later on we shall use the following

Lemma 3. Let $K \supseteq A$ be a ring extension and $\alpha \in K$ be an algebraic element over $A$ with a regular minimal polynomial $f(x) \in A[x]$. Then there exists a ring extension $K_{1} \supseteq A$ such that $Q(A) \subseteq K_{1}, f(x)$ is a minimal polynomial of some $\beta \in K_{1}$ and $A[\alpha] \cong A[\beta]$.

Proof. Let (1) be a minimal polynomial over $A$ of the element $\alpha$. If $P=$ $Q(A) \subseteq K$, then we put $K_{1}=K$ and $\beta=\alpha$. Suppose that $P \not \subset K$. Since $f(x) \in P[x]$, by Lemma 2 it follows that there exists a commutative ring extension $K_{1} \supseteq P$ such that $f(x)$ is a minimal polynomial over $P$ of some element $\beta \in K_{1}$. Since $A \subseteq P$ and $f(x) \in A[x]$, it is clear that $f(x)$ is a minimal polynomial of $\beta$ and over $A$. Now we shall prove that $A[\alpha]$ and $A[\beta]$ are isomorphic.

First we shall show that for $g(x) \in A[x]$ the conditions $g(\alpha)=0$ and $g(\beta)=0$ are equivalent. Really, since the leading coefficient $a_{0}$ is invertible 
in $P=Q(A)$, we have

$$
g(x)=f(x) q(x)+r(x), \quad \operatorname{deg} r(x)<\operatorname{deg} f(x),
$$

where the polynomials $q(x)$ and $r(x)$ are with coefficients in $P$. It is clear that there exists a power $a_{0}^{k}(k \geq 1)$ of the element $a_{0} \in A$ such that $a_{0}^{k} q(x)$ and $a_{0}^{k} r(x)$ to be elements of $A[x]$. Then

$$
a_{0}^{k} g(x)=f(x)\left[a_{0}^{k} q(x)\right]+a_{0}^{k} r(x), \quad \operatorname{deg}\left(a_{0}^{k} r(x)\right)<\operatorname{deg} f(x) .
$$

If $g(\alpha)=0$, then $a_{0}^{k} r(\alpha)=0$ and by the minimum condition of $f(x)$ we conclude that $a_{0}^{k} g(x)=f(x)\left[a_{0}^{k} q(x)\right]$. Therefore $a_{0}^{k} g(\beta)=0$ and so $g(\beta)=0$, because $a_{0}^{k}$ is an invertible element of $P$. In similar way from $g(\beta)=0$ we receive $g(\alpha)=0$. Now it is easy to verify that the map $g(\alpha) \mapsto g(\beta)$ for all $g(x) \in A[x]$ is an isomorphism between $A[\alpha]$ and $A[\beta]$, as was to be showed.

Lemma 4. Let $P=Q(A)$ be the classical ring of quotients of a commutative reduced ring $A$ and let $f(x) \in A[x]$ be a regular minimal polynomial of the algebraic element $\alpha$. Then

(i) The rings $A[\alpha]$ and $\bar{A}=A[x] /(A[x] \bigcap f(x) P[x])$ are isomorphic.

(ii) The ring $A[\alpha]$ is reduced if and only if the quotient ring $\bar{P}=P[x] / f(x) P[x]$ is reduced.

Proof. In view the preceding lemma we may assume that $Q(A) \subseteq K$ and $\alpha \in K$.

(i) The mapping $\Phi: A[x] \rightarrow A[\alpha]$, defined by $\Phi(g(x))=g(\alpha)$ for all $g(x) \in$ $A[x]$, is a homomorphism of $A[x]$ onto $A[\alpha]$ with $\operatorname{ker} \Phi=A[x] \cap f(x) P[x]$. Really, it is clear that $A[x] \cap f(x) P[x] \subseteq \operatorname{ker} \Phi$. If $g(x) \in \operatorname{ker} \Phi$, then $g(\alpha)=$ $f(\alpha)=0$. Moreover, there exist polynomials $q(x), r(x) \in P[x]$, such that

$$
g(x)=f(x) q(x)+r(x) \quad \text { and } \quad \operatorname{deg} r(x)<\operatorname{deg} f(x) .
$$

Hence it follows that $r(\alpha)=0$. Since $P=Q(A)$, for some regular element $a \in A$ we have $\varphi(x)=\operatorname{ar}(x) \in A[x]$. But $\varphi(\alpha)=0$ and $\operatorname{deg} \varphi(x)<\operatorname{deg} f(x)$ imply $\varphi(x)=0$. So we obtain $r(x)=0$ and $g(x) \in f(x) P[x]$, as was be shown.

(ii) Let $\bar{P}$ be a reduced ring. Since

$$
\bar{A}=A[x] /(A[x] \bigcap f(x) P[x]) \cong(A[x]+f(x) P[x]) / f(x) P[x] \subseteq \bar{P},
$$

so we conclude that $\bar{A}$ is a reduced ring. Now by (i) we obtain that the ring $A[\alpha]$ is reduced. Conversely, suppose that $A[\alpha]$ is reduced. If $\bar{P}$ is not reduced and $\varphi(x)+f(x) P[x]$ is its nontrivial nilpotent element, then we may assume that $0 \neq \varphi(x) \in P[x], \operatorname{deg} \varphi(x)<\operatorname{deg} f(x)$ and $\varphi^{k}(x) \in f(x) P[x]$ for some integer $k>1$. Let $a \in A$ be a nonzero regular element such that $0 \neq a \varphi(x) \in A[x]$. Then it is clear that $a \varphi(x)+A[x] \bigcap f(x) P[x]$ is a nonzero nilpotent element of $\bar{A}$. This shows that $\bar{A}$ is not reduced ring and by (i) 
we receive that $A[\alpha]$ is not reduced, which is a contradiction. So the proof is completed.

Corollary 2. If the leading coefficient of the polynomial $f(x) \in A[x]$ is an invertible element of $A$ and $f(x)$ is a minimal polynomial of $\alpha$, then the rings $A[\alpha]$ and $A[x] / f(x) A[x]$ are isomorphic.

Proof. Let $P$ be as above. Since the leading coefficient of the polynomial $f(x)$ is invertible in $A$, it is easy to verify that $f(x) A[x] \subseteq A[x] \cap f(x) P(x) \subseteq$ $f(x) A[x]$. Then the statement follows by Lemma 4(i).

\section{Simple Algebraic extensions of Reduced Rings}

Now let $A$ be a reduced commutative ring and let $f(x) \in A[x]$ be a minimal polynomial of the algebraic element $\alpha$. It is clear that $A[\alpha]$ is reduced if and only if the ring $B[\alpha]$ is reduced for every finitely generated subring $B \subseteq A$ such that $f(x) \in B[x]$. Therefore it is sufficient to find necessary and sufficient conditions $A[\alpha]$ to be reduce when $A$ is a noetherian ring. First we shall consider the case when $A$ is a field.

Recall that a field $F$ of characteristic $p \geq 0$ is said to be perfect if $p=0$, or $p>0$ and $F^{p}=F$ ([2], p. 137). So every finite field and every algebraically closed field is perfect.

If $F$ is a field and $f(x), g(x) \in F[x]$, then as ever, by $(f, g)$ we shall denote the monic greatest common divisor over $F$ of the polynomials $f(x)$ and $g(x)$. Moreover, $f(x)$ and $g(x)$ are associated if $f(x)=a g(x)$ for some non zero element $a \in F$.

Lemma 5. Let $F$ be a field of characteristic $p \geq 0$ and let $f(x)$ be a nonzero polynomial over $F$. Then the following conditions are equivalent:

(i) The quotient ring $F[x] / f(x) F[x]$ is reduced.

(ii) The polynomial $f(x)$ is a product of distinct non associated irreducible polynomials over the field of $F$.

(iii) Either $\left(f, f^{\prime}\right)=1$, or $F$ is not perfect field of characteristic $p \neq 0$ and $\left(f, f^{\prime}\right)$ is a products of distinct non associated irreducible polynomials of the form $\varphi\left(x^{p}\right) \in F[x]$.

Proof. Let $f(x)=a f_{1}^{k_{1}}(x) f_{2}^{k_{2}}(x) \cdots f_{s}^{k_{s}}(x)$ be a factorization of $f(x)$ over $F$, where $f_{1}(x), \ldots, f_{s}(x)$ are distinct non associated irreducible polynomials over $F$ and $a \in F$. Since $\left(f_{i}, f_{j}\right)=1$ for all $i \neq j$, by the Chinese theorem ([8], p.88) we have

$$
F[x] / f(x) F[x] \cong \sum_{i=1}^{s} \oplus F[x] / f_{i}^{k_{i}}(x) F[x] .
$$

It is clear that $F[x] / f(x) F[x]$ is reduced if and only if $k_{1}=k_{2}=\cdots=k_{s}=$ 1. So we obtain that (i) and (ii) are equivalent. 
Further, let $f(x)=f_{1}(x) f_{2}(x) \cdots f_{s}(x)$ be a product of distinct non associated irreducible polynomials over $F$. Denote by $g(x)=f_{1}(x) \cdots f_{k}(x)$ the product of all factors of $f(x)$, not having multiple roots. When $k=0$ we put $g(x)=1$. If $k<s$, let $d(x)=f_{k+1}(x) f_{k+2}(x) \cdots f_{s}(x)$ be the product of all factors of $f(x)$ which have multiple roots. This happens if $p>0$ and $F$ is not perfect field (see ([2] p. 138). In such case $f_{i}(x)=\varphi_{i}\left(x^{p}\right)$, where $\varphi_{i}(x) \in F[x]$ for $i=k+1, k+2, \ldots, s$. When $k=s$ we put $d(x)=1$. Thus $f(x)=g(x) d(x)$ and either $d(x)=1$, or $d(x)=\varphi\left(x^{p}\right)$ with $\varphi(x) \in F[x]$ and $\operatorname{deg} \varphi(x) \geq 1$ (see [6] p. 162). Therefore we have $d^{\prime}(x)=0$. Since $f^{\prime}(x)=g^{\prime}(x) d(x)$ and $\left(g, g^{\prime}\right)=1$, it is clear that $d(x)=\left(f, f^{\prime}\right)$. So we see that (ii) and (iii) are equivalent, as was to be shown.

As an immediate consequence we obtain

Corollary 3. Let $F$ be a field and let $f(x) \in F[x]$ be a nonzero polynomial.

(i) If $\Delta(f) \neq 0$, then the quotient ring $F[x] / f(x) F[x]$ is reduced.

(ii) If $F$ is a perfect field, then the ring $F[x] / f(x) F[x]$ is reduced if and only if $\Delta(f) \neq 0$.

Really, it is sufficient to observe that the conditions $\left(f, f^{\prime}\right)=1$ and $\Delta(f) \neq$ 0 are equivalent.

Now let $A$ be a reduced commutative ring and let $f(x) \in A[x]$ be a minimal polynomial of the algebraic element $\alpha$. As was mentioned above, it is sufficient to find necessary and sufficient conditions under which $A[\alpha]$ is reduce, when $A$ is a noetherian ring.

Theorem 1. Let $A$ be a reduced commutative noetherian ring with classical ring of quotients $P=Q(A)$ and let $\alpha$ be an algebraic element over $A$ with a minimal polynomial $f(x) \in A[x]$. If $f(x)$ is a regular polynomial over $A$, then the following statements are equivalent:

(i) The ring $A[\alpha]$ is reduced.

(ii) For every regular element $a \in A$ the polynomial af $(x)$ is not divisible by squares of polynomials over $A$ of degree $t \geq 1$.

(iii) For every minimal idempotent $e \in P$ the polynomial e $f(x)$ is a product of distinct non associated irreducible polynomials over the field $e P$.

(iv) For every minimal idempotent $e \in P$, either $\left(e f, e f^{\prime}\right)=e$, or $e P$ is a field of characteristic $p>0, e P$ is not a perfect field and $\left(e f, e f^{\prime}\right)$ is a product of distinct non associated irreducible polynomials of the form $\varphi\left(x^{p}\right) \in e P[x]$.

Proof. Suppose that $A \subseteq K$ and $\alpha \in K$. By Lemma 3, without loss of generality, we may assume that $P=Q(A) \subseteq K$. Since $A$ is a reduced commutative notherian ring, by Goldie's Theorem (see [1], Corollary 2, p. $323)$, the ring $P$ is a finite direct sum

$$
P=A_{1} \oplus A_{2} \oplus \cdots \oplus A_{k}
$$


of fields $A_{i}$ with identity elements $e_{i}(i=1, \ldots, k)$. Then $A_{i}=e_{i} P$ and it is clear that $f_{i}(x)=e_{i} f(x)$ is a minimal polynomial of $\alpha$ over the field $A_{i}$ for $i=1, \ldots, k$. Moreover,

$$
A[\alpha] \subseteq P[\alpha]=A_{1}[\alpha] \oplus A_{2}[\alpha] \oplus \cdots \oplus A_{k}[\alpha]
$$

and $P[\alpha] \cong P[x] / f(x) P[x]$. Thus, by Lemma 4(ii) we obtain that $A[\alpha]$ is reduced if and only if the rings $A_{1}[\alpha], A_{2}[\alpha], \ldots, A_{k}[\alpha]$ are reduced. Hence, by Lemma 5 we conclude that the statements (i), (iii) and (iv) are equivalent. Therefore it is sufficient to prove that (i) and (ii) are equivalent.

Really, suppose that $A[\alpha]$ is a reduced ring but $a f(x)=p^{2}(x) q(x)$ for some regular element $a \in A$, where $p(x), q(x) \in A[x]$ and $\operatorname{deg} p(x) \geq 1$. Then

$$
p(x)=e_{1} p(x)+e_{2} p(x)+\cdots+e_{k} p(x)
$$

and without loss of generality we may assume that $\operatorname{deg} e_{1} p(x) \geq 1$. Thus the equality af $(x)=p^{2}(x) q(x)$ shows that

$$
e_{1} a f(x)=\left(e_{1} p(x)\right)^{2} e_{1} q(x)
$$

and

$$
1 \leq \operatorname{deg}\left(e_{1} p(x) q(x)\right)<\operatorname{deg}\left(e_{1} a f(x)\right)=\operatorname{deg} f_{1}(x),
$$

where $f_{1}(x)=e_{1} f(x)$. Therefore, $e_{1} p(x) q(x)+f_{1}(x) A_{1}[x]$ is a nonzero nilpotent element of the quotient ring $A_{1}[x] / f_{1}(x) A_{1}[x]$. As far as $f_{1}(x)$ is a minimal polynomial of $\alpha$ over $A_{1}$, by Corollary 2 we receive that $A_{1}[\alpha]$ is not reduced, which is impossible. Conversely, if $A[\alpha]$ is not reduced ring, then $P[\alpha]$ is not reduced and without loss of generality we may assume that $A_{1}[\alpha]$ is not reduced. Then by Corollary 2 and Lemma 5 we obtain that $f_{1}(x)=p_{1}^{2}(x) q_{1}(x)$, where $p_{1}(x), q_{1}(x) \in A_{1}[x]$ and $\operatorname{deg} p_{1}(x) \geq 1$. Now we put

$$
\begin{aligned}
& p(x)=p_{1}(x)+e_{2}+\cdots+e_{k}, \\
& q(x)=q_{1}(x)+f_{2}+\cdots+f_{k}
\end{aligned}
$$

and thus we receive $f(x)=p^{2}(x) q(x)$, where $p(x), q(x) \in P[x]$ and $\operatorname{deg} p(x) \geq$ 1. Since $\mathrm{P}$ is a ring of quotients, it follows that there exist regular elements $b, c \in A$ such that $b p(x)$ and $c q(x)$ are elements of $A[x]$. Then $a=b^{2} c$ is a regular element in $A$ and $a f(x)$ is divisible by the square of $b p(x) \in A[x]$, as was to be showed.

As was mentioned above, the main result of [11] asserts that if $\Delta(f)$ is a regular element in $A$, then $A[\alpha]$ is a reduced ring. But $A[\alpha]$ may be reduced even when $\Delta(f)=0$. Indeed, let $\alpha$ be a root of the polynomial $f(x)=x^{p}-y \in A[x]$, where $A=F(y)$ is the ring of quotients of the polynomial ring $F[y]$ over a field $F$ of characteristic $p>0$. Then $\Delta(f)=0$, 
$f(x)$ is irreducible over $A$ (see [2], p. 165) and $A[\alpha]$ is reduced by Corollary 2 and Lemma 5.

We shall say that the reduced commutative $\operatorname{ring} A$ is locally perfect if for every finitely generated subring $B \subseteq A$ and every minimal idempotent $e \in Q(B)$ the field $e Q(B)$ is perfect. If the additive group of the reduced ring $A$ is either torsion free, or locally finite, then $A$ is a locally perfect ring. Thus we have the following

Corollary 4. Let $\alpha$ be an algebraic element over the commutative ring $A$ with a regular minimal polynomial $f(x) \in A[x]$ and let $\Delta(f)$ be the discriminant of $f(x)$.

(i) If $\Delta(f)$ is a regular element in $A$, then the ring $A[\alpha]$ is reduced if and only if $A$ is reduced.

(ii) If $A$ is a reduced locally perfect ring, then $A[\alpha]$ is reduced if and only if $\Delta(f)$ is a regular element in $A$.

Proof. (i) Assume that $A$ is reduced and $\Delta(f)$ is regular in $A$, but $A[\alpha]$ is not reduced. If $\beta$ is a nonzero nilpotent element of $A[\alpha]$, then let $B$ be the subring of $A$, generated by the coefficients of $\beta$ and $f(x)$. Thus $f(x) \in B[x]$ and $\beta \in B[\alpha]$. Hence by the preceding theorem it follows that for some minimal idempotent $e \in Q(B)$ the polynomial ef $f(x)$ has multiple roots and therefore $\Delta(e f)=0$. Since $\Delta(e f)=e \Delta(f)$, we obtain that the element $\Delta(f)$ is a proper divisor of zero, which is a contradiction. As far as the converse statement is trivial, the part (i) is proved.

(ii) In view of (i) it is sufficient to prove that if $A[\alpha]$ is reduced, then $\Delta(f)$ is regular. Assume for moment that $\Delta(f) a=0$ and $0 \neq a \in A$. Let $B$ be the finitely generated subring of $A$, generated by the coefficients of $f(x)$ and the element $a \in A$. Thus $f(x) \in B[x]$ and $a \in B$. Let $e_{1}, e_{2}, \ldots, e_{n}$ be a full orthogonal system minimal idempotents of $Q(B)$. Then

$$
\Delta(f)=\Delta\left(e_{1} f\right)+\Delta\left(e_{2} f\right)+\cdots+\Delta\left(e_{n} f\right),
$$

where $\Delta\left(e_{i} f\right) \in e_{i} Q(B)$ for $i=1,2, \ldots, n$. Since each $e_{i} Q(B)$ is a field and $\Delta(f)$ is a proper divisor of zero in $B$, we conclude that for some $i$ $(1 \leq i \leq n)$ we have $\Delta\left(e_{i} f\right)=0$. This implies that $e_{i} f(x)$ has multiple roots. But $e_{i} Q(B)$ is a perfect field and by Corollary 2 and Lemma 5 we obtain that $e_{i} Q(B)[\alpha]$ is not reduced and therefore $Q(B)[\alpha]$ is not reduced ring, which is a contradiction. So the proof is completed.

Let $K$ be any ring extension of the commutative ring $A$ where $K$ is not necessary commutative. If the element $\alpha \in K$ centralizes $A$, that is $\alpha . a=$ a. $\alpha$ for all $a \in A$, then we may to consider the simple commutative ring extension $A[\alpha]$. So we have the following

Corollary 5. Let $F$ be a perfect field and let $S$ be an element of the $n \times n$ matrix ring $M(n, F)$. If $F$ contains all characteristic values of $S$, then the 
ring $F[S]$ is reduced if and only if for some non-singular matrix $T \in M(n, F)$ the matrix $T S T^{-1}$ is diagonal.

Proof. By Corollary 2 and Corollary 3(ii) the ring $F[S]$ is reduced if and only if $\Delta(f) \neq 0$ where $f=f(\lambda)$ is the minimal polynomial of $S$ in $F[\lambda]$. Since $f(\lambda)$ is the last invariant factor of the characteristic matrix $S-\lambda E$ (see [5], p. 389), this condition is equivalent with the condition the Jordan's normal form of $S$ to be diagonal.

\section{Simple algebraic extensions of IRREDUCIBLE COMMUTATIVE RINGS}

In this part we shall study the problem who the ring $A[\alpha]$ contains nontrivial idempotent elements. Later on we shall say that the idempotent $E$ of the ring $A[\alpha]$ (respectively of $A[x] / f(x) A[x]$ ) is a trivial idempotent if $E$ is an element of the subring $A$ (respectively of the subring $(A+f(x) A[x]) / f(x) A[x]$ ).

As usually we shall say that the polynomial $p(x) \in A[x]$ divides the polynomial $f(x) \in A[x]$ over the ring $A$ if there exists a polynomial $q(x) \in A[x]$ such that $f(x)=p(x) q(x)$. The polynomial $p(x) \in A[x]$ is said to be a trivial divisor of $f(x)$ if $p(x)$ divides $f(x)$ and there exists an element $a \in A$ such that

$$
p(x)+f(x) A[x]=a+f(x) A[x],
$$

that is $f(x)$ divides $p(x)-a$ over $A$. For example, if $e \in A$ is a nontrivial idempotent of $A$, then every polynomial $f(x) \in A[x]$ has a trivial decomposition

$$
f(x)=[e f(x)+(1-e)][e+(1-e) f(x)] .
$$

The decomposition $f(x)=p(x) q(x)$ over $A$ is said to be nontrivial decomposition if over $A$ the polynomials $p(x)$ and $q(x)$ are nontrivial divisors of $f(x)$. Also, the decomposition $f(x)=p(x) q(x)$ is an essential decomposition over $A$ if $p(x)$ and $q(x)$ are nontrivial divisors of $f(x)$ and $\operatorname{deg} p(x)<\operatorname{deg} f(x)$, $\operatorname{deg} q(x)<\operatorname{deg} f(x)$. We shall say that the polynomial $f(x)$ is irreducible over the ring $A$ if $f(x)$ has no nontrivial decomposition over $A$.

Recall that if $F$ is a field and $\varphi(x), \psi(x) \in F[x]$, then for the greatest common divisor $(\varphi, \psi)$ there exist polynomials $u(x), v(x) \in F[x]$ such that

$$
(\varphi, \psi)=u(x) \varphi(x)+v(x) \psi(x)
$$

and $\operatorname{deg} u(x)<\operatorname{deg} \psi(x), \operatorname{deg} v(x)<\operatorname{deg} \varphi(x)$. Likewise, if $A$ is any commutative ring and $\varphi(x), \psi(x) \in A[x]$, then by Lemma 1 it follows that for the resultant $R(\varphi, \psi)$ there exist polynomials $u(x), v(x) \in A[x]$ such that

$$
R(\varphi, \psi)=u(x) \varphi(x)+v(x) \psi(x) \in A
$$

and $\operatorname{deg} u(x)<\operatorname{deg} \psi(x), \operatorname{deg} v(x)<\operatorname{deg} \varphi(x)$. From here on we shall use these facts without special stipulations. 
Let $f(x)$ be a minimal polynomial over the field $F$ of the algebraic element $\alpha$. Since the rings $F[\alpha]$ and $F[x] / f(x) F[x]$ are isomorphic, by Chain's theorem it follows that $F[\alpha]$ contains nontrivial idempotents if and only if $f(x)$ is not associated with a power of some irreducible polynomial over $F$. Now we shall prove the following lemma, which gives the idempotents of $F[\alpha]$ in explicit form.

Lemma 6. Let $\alpha$ be an algebraic element over the field $F$ with a minimal polynomial $f(x) \in F[x]$. Then

(i) The ring $F[\alpha]$ is irreducible if and only if $f(x)$ is associated with a power of an irreducible polynomial of $F[x]$.

(ii) The elements $E_{1}(\alpha)$ and $E_{2}(\alpha)$ of $F[\alpha]$ form a full orthogonal system idempotents if and only if over $F$ there exists a decomposition $f(x)=$ $\varphi(x) \psi(x)$ such that

$$
(\varphi, \psi)=u(x) \varphi(x)+v(x) \psi(x)=1,
$$

where $\operatorname{deg}(u(x) \varphi(x))<\operatorname{deg} f(x)$ and

$$
E_{1}(\alpha)=u(\alpha) \varphi(\alpha), \quad E_{2}(\alpha)=v(\alpha) \psi(\alpha) .
$$

(iii) The elements $E_{1}(\alpha)$ and $E_{2}(\alpha)$ of $F[\alpha]$ form a nontrivial full orthogonal system idempotents if and only if over $F$ there exists an essential decomposition $f(x)=\varphi(x) \psi(x)$ such that

$$
R(\varphi, \psi)=u_{1}(x) \varphi(x)+v_{1}(x) \psi(x) \neq 0
$$

and

$$
E_{1}(\alpha)=R(\varphi, \psi)^{-1} u_{1}(\alpha) \varphi(\alpha), \quad E_{2}(\alpha)=R(\varphi, \psi)^{-1} v_{1}(\alpha) \psi(\alpha) .
$$

Proof. (i) Let $F[\alpha]$ be an irreducible ring and let

$$
f(x)=a p_{1}^{k_{1}}(x) p_{2}^{k_{2}}(x) \cdots p_{s}^{k_{s}}(x)
$$

be the canonical decomposition of $f(x)$ over the field $F$. Since $F[\alpha]$ and $F[x] / f(x) F[x]$ are isomorphic rings, by the Chinese theorem we obtain that $f(x)=a p_{1}^{k_{1}}(x)$ and therefore $f(x)$ is associated with a power of irreducible polynomial over $F$. Conversely, if $f(x)$ is associated with a power of an irreducible polynomial over $F$ and $f(x)=a p^{k}(x)$, then $f(x) F[x]=p^{k}(x) F[x]$ and $p(x) F[x] / p^{k}(x) F[x]$ is a nilideal of $F[x] / p^{k}(x) F[x]$. Since

$$
\left(F[x] / p^{k}(x) F[x]\right) /\left(p(x) F[x] / p^{k}(x) F[x]\right) \cong F[x] / p(x) F[x]
$$

and $F[x] / p(x) F[x]$ is a field, by [4], Proposition 11.5.1 we conclude that (i) follows. 
(ii)Suppose that the elements $E_{1}(\alpha)$ and $E_{2}(\alpha)$ form a full orthogonal system idempotents of $F[\alpha]$. Since the rings $F[\alpha]$ and $\bar{F}[x]=F[x] / f(x) F[x]$ are isomorphic, it follows that in $\bar{F}[x]$ there exist elements

$$
\bar{E}_{1}(x)=e_{1}(x)+f(x) F[x], \quad \bar{E}_{2}(x)=e_{2}(x)+f(x) F[x]
$$

such that $\bar{E}_{1}(x)$ and $\bar{E}_{2}(x)$ form a full orthogonal system idempotents of $\bar{F}[x]$ and $e_{1}(\alpha)=E_{1}(\alpha), e_{2}(\alpha)=E_{2}(\alpha)$. Without loss of generality we may to assume that $\operatorname{deg} e_{i}(x)<\operatorname{deg} f(x)$ for $i=1,2$. Obviously, $\bar{E}_{1}(x)$ and $\bar{E}_{2}(x)$ form a full orthogonal system idempotents if and only if $e_{1}(x)+e_{2}(x)=1$ and $e_{1}(x) e_{2}(x)=f(x) q(x)$ for some $q(x) \in F[x]$. If $\bar{E}_{1}(x)$ and $\bar{E}_{2}(x)$ form a trivial system orthogonal idempotents of $\bar{F}[x]$ and $e_{1}(x)=0, e_{2}(x)=1$, then we put $\varphi(x)=f(x), \psi(x)=1$ and $u(x)=0, v(x)=1$. Suppose that $\bar{E}_{1}(x)$ and $\bar{E}_{2}(x)$ form a nontrivial system orthogonal idempotents. Since $F[x]$ is a factorial ring (see [8], p. 142), we conclude that

$$
f(x)=\varphi(x) \psi(x), \quad e_{1}(x)=u(x) \varphi(x), \quad e_{2}(x)=v(x) \psi(x),
$$

where $\varphi(x)=\left(e_{1}, f\right)$ and $\psi(x)=\left(e_{2}, f\right)$. Moreover, the polynomials $u(x)$ and $v(x)$ in $F[x]$ are uniquely determined. Since the converse statement is trivial, so (ii) is proved.

(iii) When $\bar{E}_{1}(x)$ and $\bar{E}_{2}(x)$ form a nontrivial system orthogonal idempotents of $\bar{F}[x]$, it is clear that $0<\operatorname{deg} e_{i}(x)<\operatorname{deg} f(x)$ for $i=1,2$. Thus we obtain that the decomposition $f(x)=\varphi(x) \psi(x)$ is nontrivial and therefore $\operatorname{deg} \varphi(x) \geq 1, \operatorname{deg} \psi(x) \geq 1$. As far $e_{1}(x)+e_{2}(x)=1$, we have $(\varphi, \psi)=1$ and hence we receive

$$
R(\varphi, \psi)=u_{1}(x) \varphi(x)+v_{1}(x) \psi(x) \neq 0,
$$

where, by Lemma $1, \operatorname{deg} u_{1}(x)<\operatorname{deg} \psi(x)$ and $\operatorname{deg} v_{1}(x)<\operatorname{deg} \varphi(x)$. Now it is easy to verify that $u(x)=R(\varphi, \psi)^{-1} u_{1}(x)$ and $v(x)=R(\varphi, \psi)^{-1} v_{1}(x)$. So we prove and the statement (iii).

The following lemma is an analog of the parts (ii) and (iii) of the preceding lemma for commutative artinian rings.

Lemma 7. Let $\alpha$ be an algebraic element over the reduced commutative artinian ring $A$ with a regular minimal polynomial $f(x) \in A[x]$. Then

(i) The element $E(\alpha)$ is a nontrivial idempotent in $A[\alpha]$ if and only if over $A$ there exists a nontrivial decomposition $f(x)=\varphi(x) \psi(x)$ such that

$$
u(x) \varphi(x)+v(x) \psi(x)=1
$$

for some polynomials $u(x)$ and $v(x)$ of $A[x]$, where $\operatorname{deg}(u(x) \varphi(x))<$ $\operatorname{deg} f(x)$ and $E(\alpha)=u(\alpha) \varphi(\alpha)$.

(ii) The ring $A[\alpha]$ contains nontrivial idempotents if and only if for some nonzero idempotent $e \in A$ over the ring eA there exists an essential decomposition ef $(x)=\varphi(x) \psi(x)$ such that $R(\varphi, \psi)$ is a nonzero element of $e A$. 
Proof. By Wedderburn-Artin theorem, $A=F_{1} \oplus F_{2} \oplus \cdots \oplus F_{m}$ is a finite direct sum of fields $F_{i}$ with identity elements $e_{i}(i=1, \ldots, m)$. So we have the decomposition

$$
A[\alpha]=F_{1}[\alpha] \oplus F_{2}[\alpha] \oplus \cdots \oplus F_{m}[\alpha] .
$$

(i) Suppose that $E(\alpha)$ is a nontrivial idempotent of $A[\alpha]$. Then the elements $E_{1}(\alpha)=E(\alpha)$ and $E_{2}(\alpha)=1-E(\alpha)$ have the decompositions

$$
E_{k}(\alpha)=E_{k 1}(\alpha)+E_{k 2}(\alpha)+\cdots+E_{k m}(\alpha) \quad(k=1,2),
$$

where $E_{1 i}(\alpha)$ and $E_{2 i}(\alpha)$ form a full orthogonal system idempotents of $F_{i}[\alpha]$. Obviously $f_{i}(x)=e_{i} f(x)$ is a minimal polynomial of $\alpha$ over the field $F_{i}=$ $e_{i} A$ for all $i=1,2, \ldots, m$. By Lemma 6(ii) it follows that over $F_{i}$ there exists a decomposition $e_{i} f(x)=\varphi_{i}(x) \psi_{i}(x)$ such that

$$
\left(\varphi_{i}, \psi_{i}\right)=u_{i}(x) \varphi_{i}(x)+v_{i}(x) \psi_{i}(x)=e_{i},
$$

where $\operatorname{deg}\left(u_{i}(x) \varphi_{i}(x)\right)<\operatorname{deg} f(x)$ and

$$
E_{1 i}(\alpha)=u_{i}(\alpha) \varphi_{i}(\alpha), \quad E_{2 i}(\alpha)=v_{i}(\alpha) \psi_{i}(\alpha)
$$

for $i=1,2, \ldots, m$. Then $f(x)=\varphi(x) \psi(x)$, where

$$
\begin{aligned}
& \varphi(x)=\varphi_{1}(x)+\varphi_{2}(x)+\cdots+\varphi_{m}(x), \\
& \psi(x)=\psi_{1}(x)+\psi_{2}(x)+\cdots+\psi_{m}(x) .
\end{aligned}
$$

Moreover, $u(x) \varphi(x)+v(x) \psi(x)=1$, where

$$
\begin{aligned}
& u(x)=u_{1}(x)+u_{2}(x)+\cdots+u_{m}(x), \\
& v(x)=v_{1}(x)+v_{2}(x)+\cdots+v_{m}(x) .
\end{aligned}
$$

Obviously, $\operatorname{deg}(u(x) \varphi(x))<\operatorname{deg} f(x)$ and $E(\alpha)=u(\alpha) \varphi(\alpha)$. Since the converse statement is evident, so (i) is proved.

(ii) If $E(\alpha)$ is a nontrivial idempotent of $A[\alpha]$, then again we put $E_{1}(\alpha)=$ $E(\alpha)$ and $E_{2}(\alpha)=1-E(\alpha)$. Suppose that $E_{1}(\alpha)$ and $E_{2}(\alpha)$ have the decompositions (6). Without loss of generality we may to assume that $E_{11}(\alpha)$ and $E_{21}(\alpha)$ form a full nontrivial orthogonal system idempotents of $F_{1}[\alpha]$, where $f_{1}(x)=e f(x)$ is a minimal polynomial of $\alpha$ over the field $F_{1}=e A$ and $e=e_{1}$. Then by Lemma 6(ii), over $F_{1}$ there exists an essential decomposition ef $=\varphi(x) \psi(x)$ such that $R(\varphi, \psi) \neq 0$.

Conversely, if for some idempotent $e \in A$ over the ring $e A$ there exists an essential decomposition $\operatorname{ef}(x)=\varphi(x) \psi(x)$ such that $R(\varphi, \psi) \neq 0$, we shall have the decompositions

$$
\begin{aligned}
& \varphi(x)=\varphi_{1}(x)+\varphi_{2}(x)+\cdots+\varphi_{m}(x), \\
& \psi(x)=\psi_{1}(x)+\psi_{2}(x)+\cdots+\psi_{m}(x),
\end{aligned}
$$

where $\varphi_{i}(x)=e_{i} \varphi(x)$ and $\psi_{i}(x)=e_{i} \psi(x)$ for $i=1, \ldots, m$. Since 


$$
R(\varphi, \psi)=R\left(\varphi_{1}, \psi_{1}\right)+R\left(\varphi_{2}, \psi_{2}\right)+\cdots+R\left(\varphi_{m}, \psi_{m}\right) \neq 0,
$$

it follows that for some $k(1 \leq k \leq m)$ we have $R\left(\varphi_{k}, \psi_{k}\right) \neq 0$. Then

$$
e_{k} \text { ef }(x)=e_{k} f(x)=\varphi_{k}(x) \psi_{k}(x)
$$

is an essential decomposition over the field $F_{k}$ and by Lemma 6(ii) it follows that $F_{k}[\alpha]$ contains nontrivial idempotents. Since $F_{k}[\alpha] \subseteq A[\alpha]$, we conclude that $A[\alpha]$ contains nontrivial idempotents, as was to be shoved.

Let $I$ be an ideal of $A$ and let $I[\alpha]$ be the simple algebraic extension of $I$, which is obtained by adjoining of $\alpha$ to $I$. As for $A[x] / f(x) A[x]$, we shall say that an idempotent $E$ of the ring $A[\alpha] / I[\alpha]$ is trivial if $E$ is an element of the subring $(A+I[\alpha]) / I[\alpha]$.

Lemma 8. Let $A[\alpha]$ be any simple ring extension of the commutative ring $A$ and let $I$ be a nil-ideal of $A$.

(i) All idempotents of $A[\alpha]$ are trivial if and only if all idempotents of the quotient ring $A[\alpha] / I[\alpha]$ are trivial.

(ii) If $\alpha$ is an algebraic element over the ring $A$ with a regular minimal polynomial

$$
f(x)=a_{0} x^{n}+a_{1} x^{n-1}+\cdots+a_{n-1} x+a_{n} \quad(n \geq 1),
$$

then there exists a simple algebraic extension $\bar{A}[\beta]$ of the quotient ring $\bar{A}=A / I$, such that $A[\alpha] / I[\alpha] \cong \bar{A}[\beta]$ and

$$
\bar{f}(x)=\bar{a}_{0} x^{n}+\bar{a}_{1} x^{n-1}+\cdots+\bar{a}_{n-1} x+\bar{a}_{n} \quad\left(\bar{a}_{k}=a_{k}+I\right)
$$

is a regular minimal polynomial over $\bar{A}$ of the element $\beta$.

Proof. (i) Suppose that all idempotents of $A[\alpha]$ are trivial and let $E(\alpha)=$ $u(\alpha)+I[\alpha]$ be an idempotent of $A[\alpha] / I[\alpha]$. Then $u(\alpha)^{2}-u(\alpha) \in I[\alpha]$ and, since $I[\alpha]$ is a nil-ideal of $A[\alpha]$, by ([4], Proposition 11.5.1) it follows that there exists an idempotent $e(\alpha) \in A[\alpha]$ such that $e(\alpha)-u(\alpha) \in I[\alpha]$. Therefore $E(\alpha)=e(\alpha)+I[\alpha]$ and, since all idempotents of $A[\alpha]$ are trivial, we have $e(\alpha)=e \in A$. So we conclude that all idempotents of $A[\alpha] / I[\alpha]$ are trivial. Conversely, assume that all idempotents of $A[\alpha] / I[\alpha]$ are trivial. If $e(\alpha)$ is an idempotent of $A[\alpha]$, then $e(\alpha)+I[\alpha]$ is a trivial idempotent of $A[\alpha] / I[\alpha]$ and hence for some element $a \in A$ we have $e(\alpha)+I[\alpha]=a+I[\alpha]$. Since $e(\alpha)^{2}-e(\alpha)=0$, we obtain that $a^{2}-a \in I$. Then again by ([4], Proposition 11.5.1) we obtain that there exists an idempotent $e \in A$, such that $a-e \in I$. As far $I \subseteq I[\alpha]$, we receive

$$
e(\alpha)+I[\alpha]=e+I[\alpha] .
$$

Suppose that $e(\alpha)=e+v(\alpha)$, where $v(\alpha) \in I[\alpha]$ is a nilpotent element. Then $e(\alpha) e=e+v(\alpha) e$ is an invertible element of the ring $e A[\alpha]$. But $e(\alpha) e$ is simultaneously an idempotent of $e A[\alpha]$. Thus we obtain that $e(\alpha) e=e$ and $v(\alpha) e=0$. Now $e(\alpha)(1-e)=v(\alpha)(1-e)$ is simultaneously an idempotent 
and a nilpotent element of $(1-e) A[\alpha]$. So we conclude that $v(\alpha)(1-e)=0$ and hence

$$
v(\alpha)=v(\alpha) e+v(\alpha)(1-e)=0 .
$$

Therefore $e(\alpha)=e$ is a trivial idempotent of $A[\alpha]$ and thus (i) is proved.

(ii) Obviously, $A[\alpha] / I[\alpha]=\tilde{A}[\tilde{\alpha}]$ is a simple ring extension of the subring $\tilde{A}=(A+I[\alpha]) / I[\alpha]$, obtained by adjoining of the element $\tilde{\alpha}=\alpha+I[\alpha]$ to $\tilde{A}$. Since $f(\alpha)=0$, it is clear that $\tilde{\alpha}$ is a root of

$$
\tilde{f}(x)=\tilde{a}_{0} x^{n}+\tilde{a}_{1} x^{n-1}+\cdots+\tilde{a}_{n-1} x+\tilde{a}_{n} \quad\left(\tilde{a}_{k}=a_{k}+I[\alpha]\right) .
$$

Therefore $\tilde{A}[\tilde{\alpha}]$ is a simple algebraic extension of $\tilde{A}$. If

$$
\tilde{g}(x)=\tilde{b}_{0} x^{m}+\tilde{b}_{1} x^{m-1}+\cdots+\tilde{b}_{m-1} x+\tilde{b}_{m} \quad\left(\tilde{b}_{k}=b_{k}+I[\alpha] \in \tilde{A}\right)
$$

is a minimal nonzero polynomial of $\tilde{\alpha}$ over $\tilde{A}$, then $b_{0} \notin I$ and $m \leq n$. Suppose that $m<n$. As far $\tilde{g}(\tilde{\alpha})=g(\alpha)+I[\alpha]=I[\alpha]$, we conclude that

$$
\begin{aligned}
g(\alpha) & =b_{0} \alpha^{m}+b_{1} \alpha^{m-1}+\cdots+b_{m-1} \alpha+b_{m} \\
& =c_{0} \alpha^{s}+c_{1} \alpha^{s-1}+\cdots+c_{s-1} \alpha+c_{s} \quad\left(c_{k} \in I\right),
\end{aligned}
$$

where $b_{0} \neq c_{0}$. Now we use the fact that $a_{0}$ is an invertible element in the ring of quotients $Q(A)$ and $f(\alpha)=0$. So without loss of generality we may to suppose that $s<n$ and $c_{0}, c_{1}, \ldots, c_{s} \in \mathfrak{N i l} Q(A)$. Therefore there exists a regular element $a \in A$ such that $a b_{i} \in A(i=1, \ldots, m)$ and $a c_{j} \in I$ $(j=1, \ldots, s)$. Thus we obtain that $\alpha$ is a root of a nonzero polynomial of degree $t=\min \{m, s\}<n$, which is impossible. Hence $m=n$ and $\tilde{f}(x)$ is a minimal polynomial of $\tilde{\alpha}$ over $\tilde{A}$. By a similar way we prove that $A \bigcap I[\alpha]=I$. Then it is easy to verify that $\tilde{a}_{0}$ is a regular element of $\tilde{A}$. Moreover,

$$
\tilde{A}=(A+I[\alpha]) / I[\alpha] \cong A /(A \bigcap I[\alpha])=A / I=\bar{A} .
$$

Let $\bar{f}(x)$ be a minimal polynomial of some element $\beta$ over the ring $\bar{A}$. Then the mapping $\tilde{A}[\tilde{\alpha}] \rightarrow \bar{A}[\beta]$, defined by $\tilde{\alpha} \mapsto \beta$ and $a+I[\alpha] \mapsto a+I$ for $a \in A$ is an isomorphism, as was to be showed.

Now by Lemma 8(ii) we shall prove following theorem.

Theorem 2. Let $\alpha$ be an algebraic element over an artinian commutative ring $A$ with a regular minimal polynomial $f(x) \in A[x]$. If $\bar{A}=A / \mathfrak{N i l} A$ and $\bar{f}(x)$ is the natural image of $f(x)$ into $\bar{A}[x]$, then

(i) $A[\alpha]$ is irreducible if and only if $\bar{A}$ is a field and $\bar{f}(x)$ is associated with a power of some irreducible polynomial over $\bar{A}$.

(ii) $A[\alpha]$ contains only trivial idempotents if and only if for every minimal idempotent $\bar{e} \in \bar{A}$ the polynomial $\bar{e} \bar{f}(x)$ is associated with a power of some irreducible polynomial over the field $\bar{e} \bar{A}$. 
Proof. If $A$ is an artinian commutative ring, then $\bar{A}=A / \mathfrak{N i l} A$ is a finite direct sum of fields $[6,7]$. Since $A$ is irreducible if and only if $\bar{A}$ is irreducible, by Lemma 8 we obtain that $A[\alpha]$ is irreducible if and only if $\bar{A}[\beta]$ is irreducible, where $\bar{A}$ is a field and $\bar{f}(x)$ is a regular minimal polynomial of $\beta$ over the field $\bar{A}$. Then the statement (i) follows by Lemma 7(i). Again by Lemma 8 it follows that $A[\alpha]$ contains only trivial idempotents if and only if $\bar{A}[\beta]$ contains only trivial idempotents. Since $\bar{A}$ is a finite direct sum of fields, by Lemma 7 (i) we conclude that for every minimal idempotent $\bar{e} \in \bar{A}$ the ring $\bar{e} \bar{A}[\beta]$ contains only trivial idempotents. So by Lemma 8 we obtain and the statement (ii).

Theorem 3. Let $\alpha$ be an algebraic element over a commutative noetherian ring $A$ with a monic minimal polynomial $f(x) \in A[x]$ and let $P=Q(\bar{A})$ be the ring of quotients of $\bar{A}=A / \mathfrak{N i l} A$. The ring $A[\alpha]$ contains nontrivial idempotents if and only if over the ring $\bar{P}$ there exists a nontrivial decomposition $\bar{f}(x)=\bar{\varphi}(x) \bar{\psi}(x)$ such that $\bar{u}(x) \bar{\varphi}(x)+\bar{v}(x) \bar{\psi}(x)=\overline{1}$ for some polynomials $\bar{u}(x), \bar{v}(x) \in P[x]$, where $\operatorname{deg}(\bar{u}(x) \bar{\varphi}(x))<\operatorname{deg} \bar{f}(x)$ and $\bar{u}(x) \bar{\varphi}(x) \in \bar{A}[x]$.

Proof. Suppose that $A[\alpha]$ contains a nontrivial idempotent $E(\alpha)$. Then by Lemma $8(\mathrm{i})$ it follows that $E(\alpha)+I[\alpha]$ is a nontrivial idempotent of $A[\alpha] / I[\alpha]$, where $I=\mathfrak{N i l} A$. Now by Lemma 8 (ii) we conclude that there exists a nontrivial idempotent $\bar{E}(\beta)$ of the ring $\bar{A}[\beta]$, where $\bar{f}(x) \in \bar{A}[x]$ is a minimal polynomial of $\beta$. Without loss of generality, by Lemma 3 we may assume that $\bar{E}(\beta)$ is a nontrivial idempotent of $P[\beta]$. Since $P$ is a reduced artinian ring, by Lemma 7(i) we conclude that over the ring $P$ there exists a nontrivial decomposition $\bar{f}(x)=\bar{\varphi}(x) \bar{\psi}(x)$ such that $\bar{u}(x) \bar{\varphi}(x)+\bar{v}(x) \bar{\psi}(x)=$ $\overline{1}$ for some polynomials $\bar{u}(x)$ and $\bar{v}(x)$ of $P[x]$, where $\operatorname{deg}(\bar{u}(x) \bar{\varphi}(x))<$ $\operatorname{deg} \bar{f}(x)$ and $\bar{E}(\beta)=\bar{u}(\beta) \bar{\varphi}(\beta) \in \bar{A}[\beta]$. Since $\bar{f}(x) \in \bar{A}[x]$ is a monic minimal polynomial of $\beta$ over $\bar{A}$ and $\operatorname{deg}(\bar{u}(x) \bar{\varphi}(x))<\operatorname{deg} \bar{f}(x)$, it is clear that $\bar{E}(\beta)=\bar{u}(\beta) \bar{\varphi}(\beta) \in \bar{A}[\beta]$ implies $\bar{u}(x) \bar{\varphi}(x) \in \bar{A}[x]$.

Conversely, suppose that the polynomial $f(x) \in A[x]$ satisfy the conditions of the theorem and let $g(x)$ be a polynomial in $A[x]$ such that $\bar{g}(x)=\bar{u}(x) \bar{\varphi}(x)$. If $\beta$ is an algebraic element over $\bar{A}$ with a minimal polynomial $\bar{f}(x) \in \bar{B}[x]$, then by Lemma 8 (ii) it follows that $A[\alpha] / I[\alpha]$ and $\bar{A}[\beta]$ are isomorphic rings. Since $\bar{g}(\beta)=\bar{u}(\beta) \bar{\varphi}(\beta)$ is a nontrivial idempotent in $\bar{A}[\beta]$, the element $g(\alpha)+I[\alpha]$ is a nontrivial idempotent in $A[\alpha] / I[\alpha]$ (see the proof of Lemma 8(ii)). If $u=g^{2}(\alpha)-g(\alpha)$, then by Proposition 3.6.1 [7] we conclude that $E(\alpha)=g(\alpha)-x[1-2 g(\alpha)]$ is a nontrivial idempotent of $A[\alpha]$, where

$$
x=\frac{1}{2}\left(2 u-\left(\begin{array}{l}
4 \\
2
\end{array}\right) u^{2}+\left(\begin{array}{l}
6 \\
3
\end{array}\right) u^{3}-\cdots\right) .
$$

So the theorem is proved. 
It is easy to verify that in the preceding theorem the condition $f(x)$ to be a monic polynomial is not necessary. Really, let $f(x)=4 x^{2}-1$ be a minimal polynomial of the algebraic element $\alpha$ over the integer ring $\mathbb{Z}$. Then $f(x)=(2 x-1)(2 x+1)$ is a nontrivial decomposition over the field $\mathbb{Q}=Q(\mathbb{Z})$ and $2^{-1}(2 x+1)-2^{-1}(2 x-1)=1$. Thus $e(x)=2^{-1}(2 x+1)=x+2^{-1}$ is not element of $\mathbb{Z}[x]$, but

$$
e(\alpha)=\alpha+2^{-1}=\alpha+2 \alpha^{2}
$$

is an idempotent of $\mathbb{Z}[\alpha]$.

For regular minimal polynomials we have the following

Corollary 6. Let $A$ be a commutative noetherian ring and let $P=Q(\bar{A})$ be the ring of quotients of $\bar{A}=A / \mathfrak{N i l} A$. Suppose that $f(x)$ is a regular minimal polynomial of an algebraic element $\alpha$ over the ring A. If for every minimal idempotent $e \in P$ the polynomial ef $(x)$ is associated with a power of some irreducible polynomial over the field $e P$, then all idempotents of the ring $A[\alpha]$ are trivial.

The proof of this corollary is as the proof of Theorem 3 .

\section{REFERENCES}

[1] V. A. Andrunakievič and J. M. Rjabuhin, Radikaly algebr i strukturnaya teoriya, Sovremennaya Algebra [Contemporary Algebra], "Nauka", Moscow, 1979.

[2] N. Burbaki, Algebra. Mnogochleny i polya. Uporyadochennye gruppy. Elements of Mathematics, Translated from the French by V. E. Govorov, J u. I. Manin, A. V. Mihalev, A. L. Šmelkin, Edited by Jr. I. Manin, Izdat. "Nauka", Moscow, 1965.

[3] P. M. Gudivok, V. P. Rudko, and V. A. Bovdi. Kristalografichni grupi, Uzhgorodskii Natsionalnï Universitet, Uzhgorod, 2006.

[4] F. Kasch, Moduli i koltsa, "Mir", Moscow, 1981. Translated from the German by E. N. Zaharova and M. I. Ursul, With a preface by L. A. Skornjakov.

[5] A. G. Kurosh, Lectures in general algebra, Translated by Ann Swinfen; translation edited by P. M. Cohn. International Series of Monographs in Pure and Applied Mathematics, Vol. 70. Pergamon Press, Oxford, 1965.

[6] T. Y. Lam, A first course in noncommutative rings, volume 131 of Graduate Texts in Math- ematics. Springer-Verlag, New York, 1991.

[7] J. Lambek, Lectures on rings and modules, Chelsea Publishing Co., New York, second edition, 1976.

[8] S. Lang, Algebra, Addison-Wesley Publishing Co., Inc., Reading, Mass., 1965.

[9] S. V. Mihovski, Roots of polynomials over commutative rings, Nauk. Visn. Uzhgorod. Univ. Ser. Mat. Inform., (17):161-169, 2008.

[10] S. V. Mihovski, Simple algebraic extensions of commutative rings without nilpotent or idempotent elements, C. R. Acad. Bulgare Sci., 65(12):1631-1640, 2012.

[11] N. A. Nachev, Nilpotent elements and idempotents in commutative group rings, Comm. Algebra, 33(10):3631-3637, 2005. 
[12] D. S. Passman, The algebraic structure of group rings, Pure and Applied Mathematics, Wiley-Interscience [John Wiley \& Sons], New York, 1977.

[13] B. L. van der Varden, Algebra, "Nauka", Moscow, second edition, 1979. Translated from the German by A. A. Belskii, With a foreword by Yu. I. Merzlyakov.

\section{S.V. Mihovski}

University of Plovdiv "P.Hilendarski"

TSAR Assen Str. 24

Plovdiv 4000

BUlgaria

E-mail address: mihovski@uni-plovdiv.bg 